\title{
Sistema de números reais: intuição ou rigor?
}

\author{
José C. Magossi
}

\section{Resumo}

É clássico que algumas ideias presentes em cursos de Cálculo remontam à Antiguidade, desde Arquimedes, que, entre outros cientistas, debatia também a existência do continuum. Esse problema foi abordado por I. Newton e G. W. Leibniz, os quais, no século XVII, desenvolveram o Cálculo. Mas a intuição geométrica, já no século XVIII, não dava conta de resolver problemas de "continuidade" que se apresentavam aos matemáticos. B. Bolzano, A. L. Cauchy, K. Weierstrass e R. Dedekind questionavam os fundamentos e iniciaram a era do rigor em matemática, cujo Norte acaba por ser o sistema dos números reais: um corpo ordenado completo. Um diagnóstico em livros atuais de Cálculo mostra-nos que intuição e rigor mesclam-se, seja em definições ou em demonstrações. Isso pode gerar dúvidas ao leitor menos atento. Objetiva-se expor um cenário em que a intuição geométrica revele estratégias pedagógicas e o rigor indique a necessidade da precisão matemática ${ }^{1}$.

Palavras-chave: Intuição; Rigor; Sistema de Números Reais; Teorema do Valor Médio; Teorema do Valor Intermediário.

\section{Abstract}

It is classic that some ideas present in courses of Calculus go back to Antiquity, since Archimedes, who, among other scientists, also debated the existence of the continuum. This problem was addressed by I. Newton and G. W. Leibniz, who, in the seventeenth century, developed the Calculus. But geometric intuition, as early as the eighteenth century, did not solve problems of "continuity" that presented themselves to mathematicians. B. Bolzano, A. L. Cauchy, K. Weierstrass, and R. Dedekind questioned the foundations and began the era of rigor in mathematics, whose North turns out to be the system of real numbers: a complete ordered body. A diagnosis in current books of Calculus shows us that intuition and rigor merge, whether in definitions or in demonstrations. This may create doubts for the less attentive reader. The objective is to present a scenario in which geometric intuition reveals pedagogical strategies and rigor indicates the need for mathematical precision.

Keywords: Intuition; Rigor; Real Number System; Mean Value Theorem; Intermediate Value Theorem.

\footnotetext{
${ }^{1}$ Uma pequena parte deste artigo foi apresentada no $V$ Colóquio de Matemática da Região Centro-Oeste, ocorrido no Instituto Federal de Educação, Ciência e Tecnologia de Goiás, na cidade de Goiânia, de 26 a 30 de novembro de 2018, com auxílio financeiro do Faepex-Unicamp, projeto 3082/18.
} 


\section{Introdução}

Na Grécia antiga, ao escrever Os Elementos [4], Euclides magistralmente expõe uma das mais potentes ferramentas da matemática, o sistema axiomático. Ele volta-se apenas para a geometria e exibe a ideia de que um teorema é uma asserção matemática obtida por dedução, com base em axiomas e regras lógicas de inferência. Os Elementos, de Euclides, impacta em vários setores da ciência e torna-se referência básica em matemática. Além disso a matemática grega deixa também como herança a discussão sobre as quantidades infinitamente pequenas [1], as quais, para I. Newton e G. Leibniz, revelam o Cálculo. No período do surgimento do Cálculo, a intuição geométrica tornase o patamar sob o qual as discussões matemáticas são estabelecidas. As demonstrações pautadas na intuição geométrica dão-se, com muita força, no século XVIII e início do XIX. Problemas com o continuum, convergência, séries e funções colocavam os matemáticos em um terreno desconfortável, no qual a intuição geométrica, com belas justificativas, falhava no quesito rigor matemático. Foi em meados do século XIX e início do XX que a intuição geométrica deu lugar ao sistema axiomático lógico-dedutivo, isto é, ao rigor e à análise. Com isso, teoremas que outrora eram justificados com base na intuição e geometria, doravante seriam demonstrados por um sistema axiomático cujos teoremas nada mais seriam do que aqueles obtidos por dedução lógica no sistema de números reais, isto é, num corpo ordenado completo. O Teorema do Valor Intermediário-TVI e o Teorema do Valor Médio-TVM são exemplos típicos, pois são teoremas de fácil entendimento no sentido intuitivo e geométrico, mas que precisam do sistema de números reais para serem demonstrados com rigor. Um diagnóstico em livros de Cálculo revela que axiomas de completude, importante componente do sistema de números reais, raramente são exibidos e tampouco comentários sobre o sistema axiomático são mostrados. Nos teoremas TVI e TVM, nos referidos livros, há exposições sobre as facilidades de interpretação geométrica e aplicações, mas dificilmente se diz algo sobre a dificuldade em se obter uma demonstração rigorosa, tal como sucedeu no processo histórico. Fica a impressão, para o leitor menos atento, de que o objetivo é mostrar que esses teoremas são geometricamente evidentes, o que, de certa forma, não deixa de ser um equívoco histórico. É importante, segundo O. Toeplitz [23], que se indiquem as condições nas quais um conceito surgiu, devendo-se também ser preciso com a gênese de seu surgimento. Essa precisão é importante estar presente na explicação dos TVI e TVM.

\section{Toeplitz seeks to present the great discoveries in all their drama, to let him witness the origins of the problems, concepts, and facts. But he does not want to have his method labeled "historical." The historian-the mathematical historian as well-must record all that has been, whether good or bad. I, on the contrary, want to select and utilize from mathematical history only the origins of those ideas which came to prove their value ([23], pxi $i^{2}$, Prefácio de G. Köthe, Mainz, Easter, 1949).}

A insistência na intuição e geometria, no que concerne aos teoremas TVI e TVM, caracteriza uma matemática fragmentada e desconexa, em que o sistema de números reais, por um lado, é dito ser a base do Cálculo e apresentado aos alunos já nos cursos anteriores à Universidade ${ }^{3}$, e por outro

\footnotetext{
${ }^{2}$ Toeplitz busca apresentar as grandes descobertas em todo o seu drama, para que se testemunhem as origens dos problemas, conceitos e fatos. Mas ele não quer que seu método seja rotulado de "histórico". O historiador - o historiador matemático também - deve investigar todos os fatos que ocorreram, sejam eles bons ou ruins. Eu, ao contrário, quero selecionar e utilizar da história matemática apenas as origens daquelas ideias que vieram provar o seu valor (Tradução do autor).

${ }^{3}$ Os alunos do ensino médio tomam contato com várias propriedades dos números reais, tais como ordem, estruturas algébricas etc., mas não lhes é dito sobre a construção de um número real ou sobre sua existência.
} 
permanece ausente quando da demonstração e explicação de teoremas. Por exemplo, o teorema do valor intermediário, em muitos livros de Cálculo, é exposto como se fosse um teorema cuja demonstração é autoevidente e não o oposto, uma demonstração revelada apenas por meio de uma demonstração rigorosa. Nas palavras de Jean Mawhin,

C'est Bernard BOLZANO qui le premier, en 181\%, sentit la nécessité de donner une démonstration analytique de ce résultat, considéré jusqu'alors comme "géométriquement" évident ([17], p123) ${ }^{4}$.

Com muita propriedade Felix Klein cunha uma frase para indicar a drástica mudança na forma de operar a matemática nessa época; ele diz da aritmetização da matemática [12]. A aritmética deixa de ser aplicada apenas a quantidades finitas, mas também a quantidades infinitamente pequenas (ou quantidades infinitamente grandes). É a fase da mudança da intuição e geometria para o rigor [19]. Ainda na vertente da discussão sobre intuição e geometria, sobre a inserção pedagógica de métodos intuitivos e processos de rigor, Raymond L. Wilder faz um comentário que sintetiza o seguinte questionamento: Qual é o divisor de águas entre intuição e rigor?

\begin{abstract}
Early in the nineteenth century Cauchy placed the calculus on an essentially modern basis; Abel, Gauss, and Cauchy developed a rigorous treatment of infinite series; and later in the century Weierstrass worked at the "arithmetization" of analysis. A characteristic of the latter was the freeing of analysis from the intuitive geometric type of proof so prevalent at that time. (...) We must here keep in mind, however, that to "free" a theory of certain notions does not necessarily imply that those notions forever thereafter cease to be of use. (...) We might recall here that we make similar concessions to pedagogy in the teaching of high school geometry, not striving to place it on a truly axiomatic basis but allowing the student to use "preconceived notions" such as "straight," "distance," "measure." (...) Such pedagogic devices are entirely justifiable, of course, except that it is questionable just how long the student should be kept insulated from the underlying "concessions." Certainly he should not be allowed to believed that he is receiving dogma ([24], pp200-201) ${ }^{5}$.
\end{abstract}

O objetivo deste artigo não é indicar que se devam substituir cursos de Cálculo por cursos de Análise Matemática, mas sim caracterizar que cenários matemáticos devam ser elaborados de modo que o aluno visualize o desenvolvimento da matemática e que tenha clareza dos períodos de transformação matemática. No caso deste artigo, o objetivo é indicar a relevância da passagem

\footnotetext{
${ }^{4}$ Foi Bolzano quem primeiro, em 1817, sentiu a necessidade de fornecer uma demonstração analítica desse resultado, considerado até então como "geometricamente" evidente (Tradução do autor).

${ }^{5}$ No início do século XIX Cauchy colocou o cálculo numa base essencialmente moderna; Abel, Gauss e Cauchy desenvolveram um rigoroso tratamento de séries infinitas; e, no fim do século, Weierstrass trabalhou na "aritmetização da análise". Uma característica desse último foi a libertação da análise do tipo de prova por intuição geométrica tão predominante naquela época. (...) Aqui temos que ter em mente, contudo, que a "libertação" numa teoria de certas noções não implica necessariamente que essas noções deixam de ser usadas para sempre depois disso. (...) Lembramos aqui que fazemos concessões pedagógicas semelhantes no ensino de geometria nas escolas do ensino médio, sem grandes esforços para colocá-la em uma verdadeira base axiomática, mas permitindo que o aluno utilize de "noções preconcebidas" tais como "reta," "distância," "medida." (...) Tais dispositivos pedagógicos são inteiramente justificáveis, é claro, mas é questionável quanto tempo o estudante deve ser mantido isolado dessas subjacentes concessões. Certamente, ele não deve ser levado a acreditar que está recebendo um dogma. (Tradução do autor).
} 
do período em que a intuição geométrica era imperiosa para o período em que o rigor entra em cena para resolver problemas matemáticos relacionados a uma aritmética do continuum. O fazer matemática não é simplesmente debruçar-se na elaboração de processos de dedução de teoremas com base em premissas, mas também pensar, refletir sobre questões muitas vezes tangentes aos problemas em foco, os quais podem indicar intuições que farão a diferença quando se está diante de um processo dedutivo. O fazer matemática oscila entre o pensar de forma reducionista, quando, grosso modo, o conhecimento das partes implica o conhecimento do todo, e o pensar holístico, quando o todo é mais do que o conhecimento das partes. Assim, indução, dedução e heurísticas são possíveis formas de abordar a matemática com sucesso. Uma discussão semelhante é indicada por Felix Klein ([13], p208). O estudioso, interessado ou leitor de matemática, ao tomar conhecimento dessas transformações matemáticas, pode muito bem querer se aventurar na descoberta matemática [16] e em sua investigação, seja ela heurística, indutiva ou dedutiva.

Este artigo inicia-se com uma breve exposição dos axiomas de completude, sistema de números reais, TVI e TVM. Segue-se um diagnóstico da presença ou não desses tópicos em cursos de Cálculo, corroborando a ideia de que a intuição geométrica, útil para fins pedagógicos, não deve ser confundida com rigor, essencial em processos lógicos dedutivos. Após isso, mostra-se um cenário de apresentação do Teorema do Valor Intermediário e Médio, incentivando, assim, uma matemática contextualizada e não fragmentada. Mais ainda, não há como deixar de lado a "revolução científica", no sentido de Thomas Kuhn [15], que o conceito de limites caracteriza na matemática ([9], p2) com o surgimento da era do rigor.

\section{Axiomas de Completude, Teorema do Valor Intermediário e Médio}

Em matemática há também best-sellers. É o caso do livro Foundations of Geometry, de D. Hilbert, publicado no final do século XIX [11]. Nesse livro, tal como nos Elementos de Euclides, Hilbert descreve um método axiomático moderno, que, segundo ele, deveria estabelecer um sistema de axiomas para a geometria que fosse tão simples quanto possível, e completo. Hilbert explica que a palavra "completo" indica que todos os teoremas da geometria de Euclides deveriam ser dedutíveis do conjunto de axiomas exposto no livro. À parte das discussões acerca dos fundamentos da matemática, presentes entre os interlocutores de Hilbert, o termo axioma de completude aparece a partir da segunda edição de seu livro, modificado na sétima edição, e também na versão traduzida para o francês, em 1902, para indicar a ideia de que cada intervalo na reta contém um ponto limite ([18], pp110-111, e [24], pp36-38). Assim, torna-se então possível caracterizar a reta real como sendo uma reta composta de números reais em correspondência um-a-um, ou seja, cada ponto geométrico corresponde a um número real. Segundo Hilbert, axiomas de completude auxiliam, por exemplo, na demonstração do teorema de Bolzano [21] (ou Teorema do Valor Intermediário, ([2], p138)) e do Teorema do Valor Médio, os quais, segundo H. Teismann, estão entre os quatro pilares do Cálculo (os outros dois pilares são os Teoremas do Valor Extremo e o Teorema de Darboux) ([22], p101).

Essa trajetória, desde Euclides até Hilbert, mostra a importância dos sistemas axiomáticos para o desenvolvimento da matemática, que culmina, na era do rigor, em um sistema axiomático denominado sistema de números reais, o qual é um corpo ordenado completo. Nele há propriedades de corpo, álgebra abstrata e propriedades de ordem do conjunto $\mathbb{R}$, em conexão com a propriedade de 
completude. Os axiomas de completude ${ }^{6}$, importantes para o tratamento de limites e continuidade, compreendidos apenas no final do século XIX, são condições sine qua non para o estabelecimento do rigor na Análise Matemática [2] e, consequentemente, no Cálculo ${ }^{7}$.

Na sequência, expõem-se o axioma de completude, o Teorema do Valor Intermediário e o Teorema do Valor Médio, que são, como meta deste artigo, objeto de investigação e diagnóstico em livros de Cálculo utilizados em cursos de graduação. As demonstrações, clássicas, seguem, para fins didáticos e de unificação de notação, as apresentadas em R. G. Bartle e D. R. Sherbert [2]. Assumem-se também como conhecidas as definições de continuidade, funções, gráficos, supremo, ínfimo etc., clássicas em livros de Cálculo e Análise.

Axioma do Supremo - AS. [Axioma de completude de números reais]. Cada conjunto não vazio de números reais que é limitado superiormente possui um supremo em $\mathbb{R}$ ([2], p39).

Teorema do Valor Intermediário de Bolzano - TVI. Seja $I$ um intervalo e seja $f: I \rightarrow \mathbb{R}$ uma função contínua em $I$. Se $a, b \in I$ e se $k \in \mathbb{R}$ satisfaz que $f(a)<k<f(b)$, então existe um ponto $c \in I$ entre $a$ e $b$ tal que $f(c)=k$ ([2], p138).

Teorema do Valor Médio - TVM. Supõe-se que $f$ seja uma função contínua em um intervalo fechado $I=[a, b]$, e que $f$ seja derivável no intervalo aberto $(a, b)$. Então, existe pelo menos um ponto $c \in(a, b)$ tal que

$$
f^{\prime}(c)=\frac{f(b)-f(a)}{b-a}
$$

$([2], \mathrm{p} 173)$.

\section{Diagnóstico}

Intuição ou rigor não são termos excludentes no que concerne a estratégias de ensino de Cálculo. É bem possível lançar mão da intuição geométrica com fins pedagógicos e utilizar, em momentos apropriados, o rigor matemático. Exibir um contexto claro, seja com intuição, seja com rigor, é essencial em termos didáticos. O diagnóstico que se faz em livros de Cálculo é o de que justificativas geométricas e intuitivas do TVI e TVM acabam por não indicar o real significado desses teoremas. Entende-se que se faz necessário dizer sobre axiomas de completude e sobre o método dedutivo, para que os desenvolvimentos do século XIX e início do XX possam ser compreendidos e que, consequentemente, se entenda o porquê das demonstrações. Mais ainda, a ausência de axiomas de completude em livros de Cálculo faz com que alguns conceitos matemáticos fiquem velados, por conta, principalmente, da ausência de caminhos claros nas demonstrações de teoremas no sistema de números reais.

Richard Dedekind (1831-1916) observa, em seu livro Essays on the theory of numbers - Continuity and Irrational Numbers - The Nature and Meaning of Numbers:

\footnotetext{
${ }^{6}$ No Capítulo 11 do livro Contradictions, from Consistency to Inconsistency, os autores indicam novos axiomas de completude para o sistema de números reais [20].

${ }^{7}$ Há também o Axioma da Escolha, pertencente ao domínio da Lógica, conforme citado por Jean Dieudonné ([7], p6).
} 
In discussing the notion of the approach of a variable magnitude to a fixed limiting value, and especially in proving the theorem that every magnitude which grows continually, but not beyond all limits, must certainly approach a limiting value, I had recourse to geometric evidences.(...). For myself this feeling of dissatisfaction (...). I should find a purely arithmetic and perfectly rigorous foundation for the principles of infinitesimal analysis ([6], pp1-2) $)^{8}$.

Fica clara a insatisfação de Dedekind com a aceitação de fatos óbvios na matemática sem que sejam precedidos de uma demonstração rigorosa. Essa insatisfação levou-o a elaborar um método para construir o conjunto dos números reais, hoje em dia conhecido como cortes de Dedekind ([10], p177).

Não só Dedekind, mas outros matemáticos também buscaram o rigor na matemática. B. Bolzano, em 1817, foi um dos precursores na identificação da importância dos números reais na matemática. Para ele, o TVM, geometricamente evidente, carecia de uma demonstração rigorosa ([17], p123). Mas não somente o TVM, o Teorema do Valor Intermediário também ocorre em livros de Cálculo com justificativas intuitivas, sem comentários sobre sua importância no desenvolvimento da era do rigor em matemática. No livro Analysis by Its History, E. Hairer e G. Wanner comentam sobre o Teorema do Valor Intermediário:

\section{This theorem appears geometrically evident and was used by Euler and Gauss without scruples (...) Only Bolzano found that "rein analytischer Beweis" was necessary to establish more rigor in Analysis and Algebra $([10], \text { p206 })^{9}$.}

O Teorema do Valor Intermediário ocorre, em muitos livros, sob a forma geométrica delineando apenas suas características intuitivas. É comum observar, sobre o TVI, a seguinte frase: “... é um teorema que nos diz que é possível desenhar uma função sem tirar o lápis do papel." Ou seja, fatos óbvios são elencados sem, em contrapartida, a exposição de que são teoremas que induziram a transformações na história da matemática. Como já citado, B. Bolzano foi um dos primeiros cientistas a perceber que essa intuição necessita de um tratamento mais rigoroso.

Neste artigo, escolheram-se esses dois teoremas, TVI e TVM, haja vista que, em suas definições, há um forte entendimento intuitivo e, de certa forma, sob a ótica do leitor iniciante em matemática, dispensam demonstração, pois são autoevidentes. H. Teismann [22], num artigo sobre axiomas de completude, denomina os teoremas que podem ser considerados os quatro pilares do Cálculo, a saber, Teorema do Valor Intermediário, Teorema do Valor Médio, Teorema do Valor Extremo e Teorema de Darboux.

In fact, one first-year student recently told me that her high school teacher had called these statements "silly theorems", presumably because they are so obvious/self-

\footnotetext{
${ }^{8}$ Ao discutir a noção de aproximação de uma magnitude variável para um valor de limite fixo, e especialmente ao provar o teorema de que cada magnitude que cresce continuamente, mas não além de todos os limites, deve certamente aproximar-se de um valor fixo, eu tive que recorrer a evidências geométricas (...). Para mim esse sentimento de insatisfação (...). Eu deveria achar uma base puramente aritmética e perfeitamente rigorosa dos princípios de análise infinitesimal (Tradução do autor)

${ }^{9}$ Esse teorema aparenta ser geometricamente evidente e foi utilizado por Euler e Gauss sem escrúpulos (...) Somente Bolzano entendeu que "uma Prova puramente analítica" era necessária para estabelecer mais rigor na Análise e álgebra (Tradução do autor).
} 
evident that only "silly" mathematicians would make any fuss about them ([22], p103) $)^{10}$.

A nota de rodapé “ 2 " nessa citação é a seguinte:

Most likely, referring only to the first three, as Darboux's Theorem is almost never mentioned in high school ${ }^{11}$.

No entanto, essa característica de autoevidência é exatamente o que motivou os desenvolvimentos nos fundamentos da matemática [14] de forma a se ter hoje em dia o sistema de números reais. H. Teismann completa:

\begin{abstract}
The fact that these "silly theorems" turn out to be equivalent to the completeness of the real numbers may offer a philosophical "explanation" as to why they almost seem to be too obvious. After all, the completeness of the reals itself appears completely self-evident and only becomes "a problem" when somebody (like B. Bolzano [4]) sets out to prove self-evident facts such as the Intermediate Value Theorem. In fact, Bolzano appears to have been the first to recognize the connection between the Intermediate Value Theorem and completeness. He also regarded the Intermediate Value Theorem as so self-evident that its mere confirmation would not have warranted a proof [4], ([22], p103) ${ }^{12}$.
\end{abstract}

A referência [4] nessa citação refere-se ao texto de B. Bolzano, traduzido em [21] $]^{13}$.

Não é somente o Teorema do Valor Intermediário que gera problemas com intuição e geometria; há outros, entre eles cita-se o Teorema do Valor Médio, que é também muito forte na intuição geométrica. Sobre isso, Felix Klein escreve:

\footnotetext{
${ }^{10} \mathrm{Na}$ verdade, um estudante do primeiro ano me disse recentemente que seu professor de escola secundária tinha chamado estas afirmações ${ }^{2}$ de "teoremas bobos", presumivelmente porque eles são tão óbvios/autoevidentes que somente matemáticos "bobos" fariam qualquer alvoroço sobre eles (Tradução do autor).

${ }^{11}$ Muito provavelmente, referindo-se aos três primeiros, já que o Teorema de Darboux quase nunca é mencionado no ensino médio (Tradução do autor).

${ }^{12} \mathrm{O}$ fato de esses "teoremas bobos" virem a ser equivalentes à completude de números reais pode oferecer uma "explicação" filosófica a respeito do porquê de parecerem tão óbvios. Afinal de contas, a completude dos reais parece totalmente autoevidente e só se torna "um problema" quando alguém (como B. Bolzano [4]) resolve provar fatos autoevidentes, tais como o Teorema do Valor Intermediário. Na verdade, Bolzano parece ter sido o primeiro a reconhecer a conexão entre o Teorema do Valor Intermediário e completude. Ele também considerava o Teorema do Valor Intermediário como tão autoevidente que sua mera confirmação não teria garantido uma prova [4] (Tradução do autor).

${ }^{13}[\ldots]$ while the geometrical truth to which we refer here is [...] extremely evident, and therefore needs no proof in the sense of confirmation, it nonetheless needs justification. For its component concepts are obviously so combined that one cannot hesitate for a moment to say that it is not one of those simple truths which are called basic propositions, or basic truths [...]. It is a theorem or consequent-truth [...] and, therefore, [...] it must be proved by derivation from these other truths ([22], p103) (Tradução do autor: [...] enquanto a verdade geométrica à qual nos referimos aqui é [...] extremamente evidente, e portanto, não necessita de prova no sentido de confirmação, não obstante, ela necessita de justificação. Pois seus conceitos componentes são obviamente tão combinados, que não podemos hesitar por um momento em dizer que não se trata de uma daquelas verdades simples, chamadas de proposições básicas, ou verdades básicas [...]. é um teorema ou uma verdade consequente [...] e, portanto, [...] deve ser provado por derivação, de acordo com outras verdades básicas [...].)
} 


\begin{abstract}
How can I give an exact proof of the mean value theorem, without appealing to geometric intuition? (...) For this reason such rigorous proof had to wait for Weierstrass and his followers, to whom, also, we owe the spread of the modern arithmetic concept of the number continuum ([13], p213) ${ }^{14}$.
\end{abstract}

Esse teorema, por sua vez, como descrito em [2] é um "lobo na pele de cordeiro":
In fact, the Mean Value Theorem is a wolf in sheep's clothing and is the Funda- mental Theorem of Differential Calculus ([2], p174) ${ }^{15}$.

Não se pretende, neste texto, defender ou criticar o teorema do valor médio ${ }^{16}$, mas sim indicar a pluralidade de estratégias matemáticas, pedagógicas que sejam, viáveis a uma exposição coesa. São teoremas que ocorrem em livros de Cálculo e cuja "demonstração", quando exibida, segue a intuição geométrica na maioria das vezes, ou seguem métodos dedutivos via axiomas de completude em raras ocasiões. Mais ainda, em alguns livros, "nas entrelinhas", fica subentendido que a importância desse teorema está somente em sua interpretação geométrica. Esses dizeres, que justificam a intuição e geometria, ao serem ditos aos alunos, podem não lhes mostrar a trajetória importante, de Bolzano e seus contemporâneos, por exemplo, em buscar precisão em matemática.

Da forma como intuição e geometria são apresentadas nos livros de Cálculo, diga-se de passagem, como motores propulsores, um cuidado mais intenso deve-se ter para que não se confundam a essência dos conceitos matemáticos com a facilidade que se pode ter em transmiti-los. Às vezes pode ser mais apropriado seguir o rigor do que a intuição, pois o aluno precisa saber do século XVIII, do período de descobertas, do período de inúmeras interrogações acerca de transformações matemáticas [14]. O aluno pode muito bem conhecer as trajetórias históricas que fizeram com que essa intuição "generosa", salvo olhares atentos, indicasse credibilidade nos teoremas sem questionamentos sobre rigor.

O diagnóstico ${ }^{17}$ nos livros pautou-se apenas pela presença de axiomas de completude e demonstrações dos TVI e TVM, observando a existência, ou não, da caracterização do sistema de números reais como sendo um corpo ordenado completo. Há outros pontos a serem observados que decorrem dessas mesmas observações, tais como o conceito de integrais, o conceito de funções etc., mas que não foram tratados neste texto. Além disso, o diagnóstico foi feito no momento da escrita deste artigo, não se sabe se em outros momentos esse cenário e diagnóstico poderão se alterar.

\footnotetext{
${ }^{14}$ Como podemos fornecer uma prova exata do teorema do valor médio, sem apelar para a intuição geométrica? (...) Por essa razão, essa prova tão rigorosa teve que esperar por Weierstrass e seus seguidores, aos quais, também, nós devemos a disseminação do conceito da aritmética moderna de número contínuo. (Tradução do autor).

15 De fato, o Teorema do Valor Médio é um lobo na pele de cordeiro e é o Teorema Fundamental do Cálculo Diferencial (Tradução do autor)

${ }^{16}$ Os artigos de L. Bers e L. W. Cohen, por exemplo, indicam caminhos que, segundo eles, podem ser trilhados sem um foco excessivo no teorema do valor médio [3] e [5].

${ }^{17}$ Nesse diagnóstico foram analisados livros de Cálculo (vários deles) que comumente ocorrem nas referências bibliográficas dos cursos de Cálculo no Brasil. Deve-se notar que não foi feita uma análise estatística baseada numa grande amostra de dados. Abre-se espaço, desse modo, como parte de trabalhos futuros, que uma análise estatística detalhada seja elaborada fundamentada em "todos" os livros de Cálculo publicados no Brasil e, quiçá, no exterior.
} 


\section{Cenário para uma matemática não fragmentada}

O objetivo nesta seção é expor um cenário que facilite a visualização do Cálculo (e extensivamente, a Análise Matemática) como um sistema lógico-dedutivo. O ganho dessa exposição é o de tornar-se possível analisar com mais profundidade seus teoremas e obter ferramentas matemáticas necessárias para avançar em cursos nos quais esse ferramental é pré-requisito. Além disso, abre-se espaço para que o aluno possa se aventurar na busca por novas demonstrações e novos caminhos para a obtenção da demonstração desses teoremas (e possivelmente de outros). Com isso, estimase que seja possível uniformizar a relação intuição-rigor. Há condições, desse modo, para que estratégias pedagógicas sejam elaboradas com vistas ao rigor e à intuição. Na sequência, expõe-se um cenário que identifica o sistema de números reais como sendo um corpo ordenado completo, no qual os axiomas de completude exercem um papel importante na caracterização dos processos lógico-dedutivos. Nesta seção escolhem-se dois teoremas para fazer parte desse cenário: o Teorema do Valor Intermediário e o Teorema do Valor Médio. Esses são dois teoremas que, segundo o diagnóstico na seção anterior, são expostos nos livros levando em conta, na maioria das vezes, apenas a intuição geométrica. Ou seja, leva-se em conta o óbvio, conforme relatos obtidos em estudos com foco na gênese da descoberta desses conceitos. O que se expõe nos livros, com fins didáticos, acredita-se, é de certa forma apenas uma pequena parte dos problemas e "angústias" matemáticas que marcaram determinadas épocas. A exposição ipsis litteris desses conceitos e descobertas matemáticas pode ser útil sob a ótica de estudos da História da Matemática (ou História da Ciência) e também da Epistemologia, mas não sob a ótica da busca de ferramental matemático necessário ao fazer matemática e aos aspectos pedagógicos. Ou seja, é preciso refletir sobre o melhor cenário de exposição, se o objetivo é pedagógico, ou se é fazer matemática.

O cenário ora exposto leva em conta os axiomas de corpo e de ordem, bem como o axioma de completude, clássico em textos de Análise, popularmente conhecido como axioma do supremo. A demonstração de apenas alguns teoremas é feita, principalmente, daqueles teoremas que compõem a demonstração do Teorema do Valor Intermediário e do Teorema do Valor Médio.

\subsection{Teoremas relacionados ao TVI e ao TVM}

Nesta seção, exibem-se os teoremas que serão utilizados nas demonstrações do TVI e do TVM. Por serem clássicos, apenas para alguns deles as demonstrações serão feitas (uma adaptação, em alguns casos, daquelas expostas em [2]). Para os outros teoremas seguem as demonstrações contidas no excelente livro Introduction to Real Analysis de Robert G. Bartle e Donald R. Sherbert. Assumese, como hipótese, o sistema de números reais, tal como exposto em [2]. O objetivo principal é mostrar a dependência de teoremas, mostrar o processo dedutivo que é utilizado para compor as demonstrações dos TVI e TVM. Não é objetivo expor, tal como está escrito, a sequência completa de formação dos Teoremas do Valor Intermediário e Valor Médio, mas sim alguns teoremas, os mais significativos para a composição do TVI e do TVM.

Axioma 1. Axioma do Supremo - $\boldsymbol{A} \boldsymbol{S}$ [Axioma de completude de números reais]. Cada conjunto não vazio de números reais que é limitado superiormente possui um supremo em $\mathbb{R}$ ([2], p39).

Teorema 1 (Propriedade de Arquimedes). ([2], p42). Se $x \in \mathbb{R}$, então existe $n_{x} \in \mathbb{N}$ tal que $x<n_{x}$ 
Teorema 2 (Propriedade dos Intervalos Encaixantes - IE). ([2], p48). Se $I_{n}=\left[a_{n}, b_{n}\right]$ para $n \in \mathbb{N}$ é uma sequência encaixante de intervalos fechados e limitados, então existe um número $\xi \in \mathbb{R}$ tal que $\xi \in I_{n}$, para todo $n \in \mathbb{N}$.

Demonstração. Desde que os intervalos são encaixantes, tem-se que $I_{n} \subseteq I_{1}$ para todo $n \in \mathbb{N}$ e também que $a_{n} \leq b_{1}$ para todo $n \in \mathbb{N}$. Portanto, o conjunto de números reais não vazio $A=$ $\left\{a_{n} \mid n \in \mathbb{N}\right\}$ é limitado superiormente, e assim existe, pelo axioma de completude (axioma do supremo), um número real $\xi=\sup (A)$. É óbvio que $a_{n} \leq \xi$ para todo $n \in \mathbb{N}$. Mostra-se que $\xi \leq b_{n}$ para todo $n \in \mathbb{N}$. O objetivo é mostrar que para qualquer $n$ em particular, o número $b_{n}$ é um limitante superior para o conjunto $\left\{a_{k} \mid k \in \mathbb{N}\right\}$. Para isso consideram-se os casos:

i) Se $n \leq k$, então desde que $I_{k} \subseteq I_{n}$, tem-se que $a_{k} \leq b_{k} \leq b_{n}$.

ii) Se $k<n$, então desde $I_{n} \subseteq I_{k}$, tem-se que $a_{k} \leq a_{n} \leq b_{n}$.

Assim, conclui-se que $a_{k} \leq b_{n}$ para todo $k$, assim como $b_{n}$ é um limitante superior do conjunto $\left\{a_{k} \mid k \in \mathbb{N}\right\}$. Portanto, $\xi \leq b_{n}$ para cada $n \in \mathbb{N}$. Desde que $a_{n} \leq \xi \leq b_{n}$ para todo $n \in \mathbb{N}$, tem-se que $\xi \in I_{n}$ para todo $n \in \mathbb{N}$.

Teorema 3. ([2], p63). Uma sequência convergente de números reais é limitada.

Teorema 4. ([2], p64). (a) Sejam $X=\left(x_{n}\right)$ e $Y=\left(y_{n}\right)$ sequências de números reais que convergem para $x$ e $y$, respectivamente, e seja $c \in \mathbb{R}$. Então, as sequências $X+Y, X-Y, X . Y$ e $c X$ convergem para $x+y, x-y, x . y$ e cx, respectivamente. (b) Se $X=\left(x_{n}\right)$ converge para $x$ e $Z=\left(z_{n}\right)$, é uma sequência não nula de números reais que converge para $z$ e se $z \neq 0$, então a sequência quociente $\frac{X}{Z}$ converge para $\frac{x}{z}$.

Teorema 5. ([2], p65). Se $X=\left(x_{n}\right)$ é uma sequência convergente de números reais e se $x_{n} \geq 0$ para todo $n \in \mathbb{N}$, então $x=\lim \left(x_{n}\right) \geq 0$.

Teorema 6. ([2], p65). Se $X=\left(x_{n}\right)$ e $Y=\left(y_{n}\right)$ são sequências convergentes de números reais e se $x_{n} \leq y_{n}$ para todo $n \in \mathbb{N}$, então $\lim \left(x_{n}\right) \leq \lim \left(y_{n}\right)$.

Teorema 7. ([2], p66). Se $X=\left(x_{n}\right)$ é uma sequência convergente de números reais e se a $\leq$ $x_{n} \leq b$ para todo $n \in \mathbb{N}$, então $a \leq \lim \left(x_{n}\right) \leq b$.

Teorema 8. ([2], p66). Sejam $X=\left(x_{n}\right), Y=\left(y_{n}\right)$ e $Z=\left(z_{n}\right)$ sequências de números reais tais que $x_{n} \leq y_{n} \leq z_{n}$ para todo $n \in \mathbb{N}$, e que $\lim \left(x_{n}\right)=\lim \left(z_{n}\right)$. Então $Y=\left(y_{n}\right)$ é convergente $e$ $\lim \left(x_{n}\right)=\lim \left(y_{n}\right)=\lim \left(z_{n}\right)$.

Teorema 9 (Teorema da Convergência Monótona). ([2], pp71-72). Uma sequência monótona de números reais é convergente se e somente se ela é limitada. Além disso:

a) Se $X=\left(x_{n}\right)$ é uma sequência limitada crescente então $\lim \left(x_{n}\right)=\sup \left\{x_{n} \mid n \in \mathbb{N}\right\}$.

b) Se $Y=\left(y_{n}\right)$ é uma sequência limitada decrescente então $\lim \left(y_{n}\right)=\inf \left\{y_{n} \mid n \in \mathbb{N}\right\}$.

Teorema 10 (Teorema da Subsequência Monótona). ([2], p80). Se $X=\left(x_{n}\right)$ é um sequência de números reais, então existe uma subsequência de $X$ que é monótona. 
Teorema 11 (Teorema de Bolzano-Weierstrass). ([2], p81). Uma sequência limitada de números reais tem uma subsequência convergente.

Teorema 12. ([2], p115). Seja $A \subseteq \mathbb{R}$, seja $f: A \rightarrow \mathbb{R}$ e seja $c \in \mathbb{R}$ um ponto de acumulação de A. $S e$

$$
\left.\lim _{x \rightarrow c} f>0 \quad \text { [respectivamente, } \quad \lim _{x \rightarrow c} f<0\right],
$$

então existe uma vizinhança $V_{\delta}(c)$ de c tal que $f(x)>0$ /respectivamente, $f(x)<0$ / para todo $x \in A \cap V_{\delta}(c) \operatorname{com} x \neq c$.

Teorema 13 (Teorema do limitante). ([2], p135). Seja $I=[a, b]$ um intervalo fechado e limitado e seja $f: I \rightarrow \mathbb{R}$ uma função contínua em $I$. Então $f$ é limitada em $I$.

Teorema 14 (Teorema do máximo e mínimo). ([2], p136). Seja $I=[a, b]$ um intervalo fechado e limitado e seja $f: I \rightarrow \mathbb{R}$ uma função contínua em $I$. Então $f$ tem um máximo absoluto e um minimo absoluto em $I$.

Teorema 15 (Teorema de Localização de Raízes - LR). ([2], p137). Seja $I=[a, b]$ e seja $f: I \rightarrow \mathbb{R}$ uma função contínua em I. Se $f(a)<0<f(b)$, ou se $f(a)>0>f(b)$, então existe um número $c \in(a, b)$ tal que $f(c)=0$.

Demonstração. Assume-se que $f(a)<0<f(b)$. Gera-se uma sequência de intervalos por meio de sucessivas bissecções. Seja $I_{1}=\left[a_{1}, b_{1}\right]$, onde $a_{1}=a$ e $b_{1}=b \operatorname{com} p_{1}=\frac{a_{1}+b_{1}}{2}$ o ponto médio de $I_{1}$. Se $f\left(p_{1}\right)=0$ então toma-se $c=p_{1}$ e o teorema está provado. Se $f\left(p_{1}\right) \neq 0$, então ou $f\left(p_{1}\right)>0$ ou $f\left(p_{1}\right)<0$. Se $f\left(p_{1}\right)>0$, então faz-se $a_{2}=a_{1}, b_{2}=p_{1}$, enquanto se $f\left(p_{1}\right)<0$, então faz-se $a_{2}=p_{1}$ e $b_{2}=b_{1}$. Em quaisquer casos, faz-se $I_{2}=\left[a_{2}, b_{2}\right]$, e tem-se $I_{2} \subset I_{1}$ e $f\left(a_{2}\right)<0$ e $f\left(b_{2}\right)>0$. Continua-se esse processo de bissecção. Supõe-se que os intervalos $I_{1}, I_{2}, \ldots, I_{k}$ tenham sido obtidos de modo análogo, por sucessivas bissecções. Então, tem-se que $f\left(a_{k}\right)<0$ e $f\left(b_{k}\right)>0$, e fazemos $p_{k}=\frac{a_{k}+b_{k}}{2}$. Se $f\left(p_{k}\right)=0$, toma-se $c=p_{k}$ e o processo termina. Se $f\left(p_{k}\right)>0$, então seja $a_{k+1}=a_{k}$ e $b_{k+1}=p_{k}$, enquanto se $f\left(p_{k}\right)<0$, seja $a_{k+1}=p_{k}$ e $b_{k+1}=b_{k}$. Em quaisquer casos, faz-se $I_{k+1}=\left[a_{k+1}, b_{k+1}\right]$; então $I_{k+1} \subset I_{k}$ e $f\left(a_{k+1}\right)<0$ e $f\left(b_{k+1}\right)>0$.

Se o processo terminar com um ponto $p_{n}$ tal que $f\left(p_{n}\right)=0$, então o processo termina. Se o processo não terminar, então obtém-se uma sequência de intervalos encaixantes limitada e fechada $I_{n}=\left[a_{n}, b_{n}\right]$, tal que para cada $n \in \mathbb{N}$ se tem

$$
f\left(a_{n}\right)<0 \text { e } f\left(b_{n}\right)>0 .
$$

Além disso, desde que os intervalos são obtidos por repetidas bissecções, o comprimento de $I_{n}$ é igual a $b_{n}-a_{n}=\frac{b-a}{2^{n-1}}$. Isso segue da Propriedade de Intervalos Encaixantes - IE que existe um ponto $c \in I_{n}$ para todo $n \in \mathbb{N}$. Desde que $a_{n} \leq c \leq b_{n}$, para todo $n \in \mathbb{N}$ e que $\lim _{n \rightarrow \infty}\left(b_{n}-a_{n}\right)=0$, segue que $\lim _{n \rightarrow \infty} a_{n}=c=\lim _{n \rightarrow \infty} b_{n}$. Desde que $f$ é uma função contínua em $x=c$, tem-se que:

$$
\lim _{n \rightarrow \infty} f\left(a_{n}\right)=f(c)=\lim _{n \rightarrow \infty} f\left(b_{n}\right) .
$$

O fato de que $f\left(a_{n}\right)<0$ para todo $n \in \mathbb{N}$ implica que

$$
f(c)=\lim _{n \rightarrow \infty}\left(f\left(a_{n}\right)\right) \leq 0 .
$$


Também, o fato de que $f\left(b_{n}\right)>0$ para todo $n \in \mathbb{N}$ implica que

$$
f(c)=\lim _{n \rightarrow \infty}\left(f\left(b_{n}\right)\right) \geq 0 .
$$

Portanto, conclui-se que $f(c)=0$. Consequentemente, $c$ é uma raiz de $f$.

Teorema 16 (Teorema do Valor Intermediário de Bolzano - TVI). ([2], p138). Seja I um intervalo e seja $f: I \rightarrow \mathbb{R}$ uma função contínua em $I$. Se $a, b \in I$ e se $k \in \mathbb{R}$ satisfaz que $f(a)<k<f(b)$, então existe um ponto $c \in I$ entre a e $b$ tal que $f(c)=k$.

Demonstração. Suponha que $a<b$ e que $g(x)=f(x)-k$. Assim, $g(a)<0<g(b)$. Pelo teorema de Localização de Raízes - LR, existe um ponto $c$ com $a<c<b$ tal que $0=g(c)=f(c)-k$. Portanto $f(c)=k$. Se $b<a$, então seja $h(x)=k-f(x)$ tal que $h(b)<0<h(a)$. Portanto existe um ponto $c$ com $b<c<a$ tal que $0=h(c)=k-f(c), \operatorname{logo} f(c)=k$.

Teorema 17 (Teorema do interior extremo). ([2], p172). Seja c um ponto interior de um intervalo $I=[a, b]$ no qual $f: I \rightarrow \mathbb{R}$ tem um extremo relativo. Se a derivada de $f$ em c existe, então $f^{\prime}(c)=0$.

Teorema 18 (Teorema de Rolle). ([2], p172). Suponha que f é uma função contínua em um intervalo fechado $I=[a, b]$, que a derivada $f^{\prime}$ existe em cada ponto do intervalo aberto $(a, b)$ e que $f(a)=f(b)=0$. Então existe pelo menos um ponto $c \in(a, b)$ tal que $f^{\prime}(c)=0$.

Teorema 19 (Teorema do Valor Médio). ([2], p173). Suponha que f seja uma função contínua em um intervalo fechado $I=[a, b]$, e que $f$ seja derivável no intervalo aberto $(a, b)$. Então, existe pelo menos um ponto $c \in(a, b)$ tal que

$$
f^{\prime}(c)=\frac{f(b)-f(a)}{b-a} .
$$

Demonstração. Considera-se a função $\phi$ definida em $I$ da seguinte forma:

$$
\phi(x)=f(x)-f(a)-\frac{f(b)-f(a)}{b-a}(x-a) .
$$

A hipótese do teorema de Rolle é satisfeita por $\phi$, uma vez que $\phi$ é contínua em $[a, b]$, diferenciável em $(a, b)$ e $\phi(a)=\phi(b)=0$. Portanto, existe um ponto $c \in(a, b)$ tal que

$$
0=\phi^{\prime}(c)=f^{\prime}(c)-\frac{f(b)-f(a)}{b-a} .
$$

Portanto, $f(b)-f(a)=f^{\prime}(c)(b-a)$.

O objetivo principal nesta seção é expor um cenário, associado ao TVI e ao TVM, para mostrar que o Cálculo pode ser visto também como um sistema lógico-dedutivo, sem se perder em excessos de rigor. Abre-se espaço, desse modo, para que estratégias pedagógicas sejam elaboradas com vistas ao rigor e à intuição. As seguintes árvores de dependência $(A \rightarrow B$ significa que na demonstração de $B$ utiliza-se de $A$ ) expõem a complexidade de teoremas, vistos como evidentes, mas cuja demonstração exige o conhecimento do sistema de números reais. 
Cenário para o TVI. Uma árvore de formação para o Teorema do Valor Intermediário.

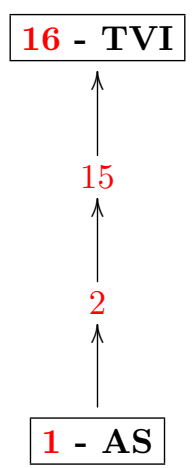

Cenário para o TVM. Uma árvore de formação para o teorema do valor médio.

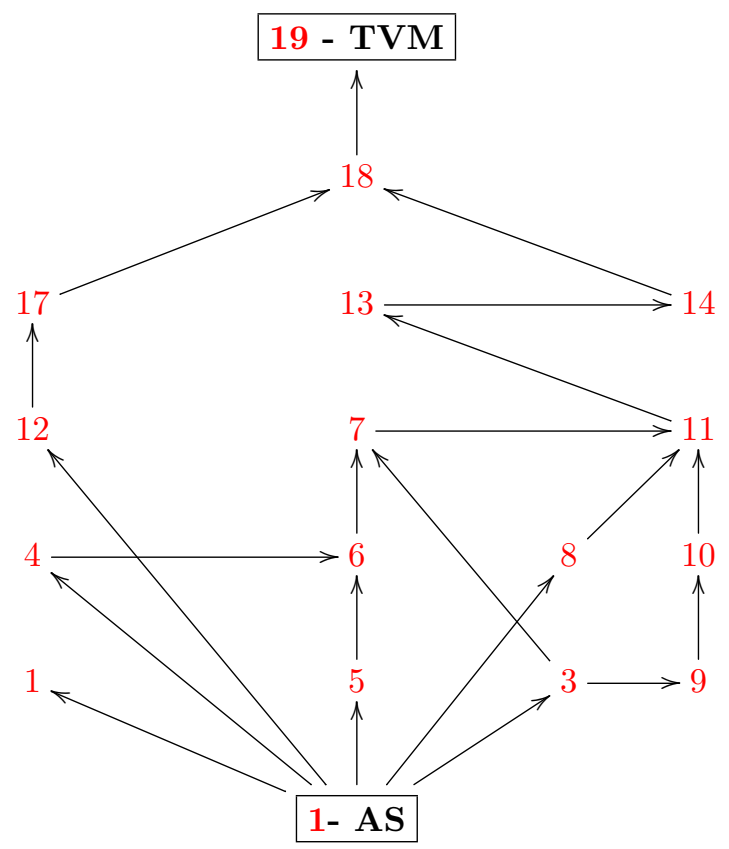

A árvore de formação do TVM é uma das inúmeras possibilidades de dedução desse teorema, que segue, neste texto, com numeração própria, os teoremas apresentados em [2]. Ao se alterarem os axiomas de completude, alteram-se as dependências dos teoremas que funcionam como nós na árvore. Qual é a árvore mais "econômica" com que se pode atingir o TVI, ou o TVM? Inúmeras perguntas podem ser elaboradas pelos alunos, e todas elas acabam por se relacionar ao real processo histórico em que se tornam claros a gênese do surgimento do conceito e seu processo de desenvolvimento histórico. É importante notar que cabe ao docente a escolha da estratégia pedagógica a ser adotada em cursos de Cálculo. A exposição de cenários em que leva em conta a essência da construção de conceitos (e das demonstrações de teoremas) não implica que o aluno 
está diante de um curso de Análise Matemática. O que se propõe com as discussões acima é simplesmente exibir cenários, contextos, em que esteja claro o porquê do surgimento de um conceito matemático, o porquê das demonstrações de teoremas, e, por conseguinte, o porquê dos processos de transformação. A escolha entre "exibir", "indicar" ou "demonstrar" faz parte do cenário, diga-se contexto, escolhido para compor uma aula de Cálculo.

\section{Conclusão}

Exibiu-se uma discussão acerca do sistema de números reais utilizados nos cursos de Cálculo. Defende-se a importância da intuição geométrica, seja como meio de desenvolvimento matemático, seja como estratégia pedagógica. No entanto, deve-se ter clareza na distinção entre a intuição $e$ geometria como parte de interrogações históricas, e intuição e geometria como parte de estratégias pedagógicas. Isso é tão importante quanto diferenciar uma demonstração matemática (uma dedução num sistema axiomático) de sua contraparte intuitiva, sem rigor matemático. Falta de clareza implica possíveis erros de compreensão de conceitos e possíveis olhares para o Cálculo (e Análise Matemática) como um amontoado de teoremas sem conexão. O exemplo da árvore de formação do TVI e do TVM mostra essa perspectiva. Intuição ou rigor? Essa é a questão deste artigo.

Para levantar questionamentos, invocam-se os Teoremas do Valor Intermediário e do Valor Médio, ambos com forte apelo intuitivo, os quais facilmente percorrem o caminho de justificativas óbvias em detrimento de demonstrações. Propõe-se que o sistema de números reais seja exposto nos livros de Cálculo, não com o objetivo de se trabalhar num sistema preciso de demonstrações tal como a Análise Matemática, mas sim com o objetivo de indicar os problemas que levaram anos para ser resolvidos, os quais, se encarados apenas com intuição e geometria, perdem a razão da existência. Dois cenários são expostos relacionados aos dois teoremas TVI e TVM para mostrar que ambos advêm de outros teoremas por meio de um sistema dedutivo, inseridos no sistema de números reais. Com isso, estima-se que os leitores possam absorver com mais facilidade a ideia de que os axiomas de completude servem para caracterizar a existência do sistema de números reais.

É claro que essa existência parte do pressuposto da aceitação, de alguma forma, da ideia de infinitésimos, da ideia de uma nova aritmética, tal como indicado por Felix Klein [12]. Sobre infinitésimos, talvez seja interessante refletir sobre os dizeres de Felix Klein [13] a respeito do livro Lehrbuch der Experimentalphysik, ${ }^{18}$ do autor Adolph Wüllner (1907).

I urge you to read this introduction with the eye of the mathematician and to reflect upon the absurdity of suppressing infinitesimal calculus in the schools because it is too difficult (...)([13], p217). ${ }^{19}$

Cabe a cada professor fazer a escolha pedagógica apropriada. Não se defende neste texto que os cursos de Cálculo transformem-se em cursos de Análise Matemática, mas que sejam indicados aos alunos os processos transformadores da matemática, os quais acarretam desenvolvimentos matemáticos e tecnológicos.

What is the calculus? (...). The calculus can be fruitfully viewed and effectively taught as a set of intuitively understood problem-solving techniques, widely appli-

\footnotetext{
${ }^{18}$ Manual de Física Experimental (Tradução do autor).

${ }^{19} \mathrm{Eu}$ insisto que vocês leiam esta introdução com o olhar do matemático e reflitam sobre o absurdo de suprimir o cálculo infinitesimal nas escolas porque ele é muito difícil (...)(Tradução do autor).
} 
cable to geometry and to physical systems. (...)The calculus is something else as well, however: a set of theorems, based on precise definitions, about limits, continuity, séries, derivatives, and integrals. (...) These two different aspects-use and justification- of the calculus, simultaneously coexisting in the modern subject, are in fact the legacies of two different historical periods: the eighteenth and the nineteenth centuries ([9], p1). ${ }^{20}$

Neste texto, indicou-se apenas um cenário entre os muitos possíveis acerca da discussão sobre sistema dedutivo. O objetivo é exatamente o incentivo aos processos de dedução que de certa forma constroem a análise matemática. Há outros exemplos de processos de transformação semelhantes ao exposto neste texto. Pode-se citar o caso do desenvolvimento das integrais de Henstock-Kurzweil, em que, a partir de "simples" alterações na definição das partições em somas de Riemann, obtém-se uma nova definição de integrais, generalizadas, que englobam uma classe muito maior de funções integráveis [8].

\section{Referências}

[1] Alexander, Amir. Infinitesimal - A teoria matemática que mudou o mundo. Jorge Zahar Editor Ltda, Rio de Janeiro, 2014.

[2] Bartle, Robert. G.; Sherbert, Donald R. Introduction to Real Analysis, Fourth Edition, Wiley India Edition, New Delhi, 2011.

[3] Bers, L. On Avoiding the Mean Value Theorem.American Mathematical Monthly 74, 5, 583, 1967.

[4] Bicudo, Irineu. Os Elementos - Euclides. Tradução de Irineu Bicudo. Editora Unesp, Rio Claro, 2009.

[5] Cohen, L.W. On being mean to the Mean Value Theorem. American Mathematical Monthly, 74 (5), 581-582, 1967.

[6] Dedekind, Richard. Essays on the theory of numbers-Continuity and Irrational Numbers-The Nature and Meaning of Numbers, The Open Court Publishing Company, 1901 (first publication). Dover Publications, 1963, 2010.

[7] Dieudonné, Jean. Foundations of Modern Analysis, Academic Press, 1960, 1969.

[8] Gordon, Russell A. The Integrals of Lebesgue, Denjoy, Perron, and Henstock. No. 4. American Mathematical Society, 1994.

[9] Grabiner, Judith V. The Origin of Cauchy's Rigorous Calculus. Dover Publications, Inc., New York, 1981.

[10] Hairer, E.; Wanner, G. Analysis by Its History. Springer-Verlag, New York, 1996.

[11] Hilbert, David. Grundlagen der Geometrie. Festschrift zur Feier der Enthullung des GaussWeber-Denkmals in Gottingen, Teubner, Leipzig, 1899. Tradução para o inglês por E. J. Townsend, PH.D.: The Foundations of Geometry, Open Court, Chicago, 1902.

${ }^{20} \mathrm{O}$ que é o cálculo? (...). O cálculo pode ser proveitosamente visto e efetivamente ensinado como um conjunto de técnicas para resolver problemas intuitivamente compreensíveis, amplamente aplicáveis à geometria e a sistemas físicos. (...) O cálculo é também, no entanto, alguma coisa diferente: um conjunto de teoremas, baseado em definições precisas, sobre limites, continuidade, séries, derivadas e integrais. (...) Esses dois aspectos diferentes do cálculo - uso e justificação -, simultaneamente coexistindo nos assuntos modernos, são de fato dois legados de dois diferentes períodos históricos: os séculos XVIII e XIX. (Tradução do autor). 
[12] Klein, Felix. Ueber Arithmetisirung der Mathematik. Göttinger Nachrichten (Geschäftliche Mittheilungen), 1895, p82. (Miss Maddison's translation in the Bulletin, 2d series, vol. 2, p241, 1896), 1895.

[13] Klein, Felix. Elementary Mathematics from an Advanced Standpoint. Geometry. Dover Publications, New York, 2004.

[14] Kline, Morris. Les fondements des mathématiques. La recherche, v. 54, pp200-208, 1975.

[15] Kuhn, Thomas. A Estrutura das Revoluções Científicas. Editora Perspectiva, São Paulo, 1994.

[16] Lakatos, Imre. Proofs and Refutations - The Logic of Mathematical Discovery. Cambridge University Press, 1976.

[17] Mawhin, Jean. Analyse: fondements, techniques, évolution. De Boeck Université, Brussels, 1992. Second edition, 1997.

[18] Moore, G. H. A house divided against itself: the emergence of first-order logic as the basis for mathematics. In: Studies in the History of Mathematics. Mathematical Association of America, pp98-136, New York, 1987.

[19] Pierpont, James. On the arithmetization of mathematics. Bulletin of the American Mathematical Society, v. 5, n. 8, pp394-406, 1899.

[20] Rioul, Olivier; Magossi, José Carlos. A Local-Global Principle for the Real Continuum. Studia Logica, Vol. 47, Capítulo 11, pp213-240. Special edition of Trends in Logic 47: Contradictions, from Consistency to Inconsistency. Editores: Prof. Walter Carnielli e Prof. Jacek Malinowski, Springer International Publishing, 2018.

[21] Russ, S. B. A translation of Bolzano's paper on the Intermediate Value Theorem. Historia Mathematica, v.7, n.2, pp156-185, 1980.

[22] Teismann, Holger. Toward a More Complete List of Completeness Axioms. Mathematical Association of America, The American Mathematical Monthly, Vol. 120, no. 2, February, pp99114, 2013.

[23] Toeplitz, Otto. The Calculus - A Genetic Approach. Chicago University Press, Chicago, 2007.

[24] Wilder, Raymond L. Introduction to The Foundations of Mathematics. (First Edition, Wiley, 1952). Dover Publications, Second Edition, New York, 2012.

José C. Magossi

Faculdade de Tecnologia - FT - Unicamp $<$ magossi@ft.unicamp.br>

Recebido: 18/03/2019 